\title{
Entering Watch Dogs*: Evaluating Privacy Risks Against Large-Scale Facial Search and Data Collection
}

\author{
Bahadır Durmaz \\ Department of Computer Science \\ Bilkent University \\ Ankara, Turkey \\ bdurmazse@gmail.com
}

\author{
Erman Ayday \\ Department of Computer and Data Sciences \\ Case Western Reserve University \\ Cleveland, $\mathrm{OH}$, USA \\ exa208@case.edu
}

\begin{abstract}
Discovering friends on online platforms have become relatively easier with the introduction of contact discovery and ability to search using phone numbers. Such features conveniently connect users by acting as unique tokens across platforms, as opposed to other attributes, such as user names. Using this feature, in this work, one of our contributions is to explore how an attacker can easily create a massive dataset of individuals residing in a given region (e.g., country) that includes high amount of personal information about such individuals. To identify the active social network accounts of individuals in a given region, we show that brute force phone number verification is possible in popular online services, such as WhatsApp, Facebook Messenger, and Twitter. We also go beyond and show the feasibility of collecting several data points on discovered accounts, including multiple facial data belonging to each account owner along with 23 other attributes. Then, as our main contribution, we quantify the privacy risk for an attacker linking a total stranger (e.g., someone it randomly comes across in public) to one of the collected records via facial features. Our results show that accurate facial search is possible in the constructed dataset and that an attacker can link a randomly taken photo (i.e., a single facial photo) of an individual to their profile with $67 \%$ accuracy. This means that an attacker can, on a large scale, create a search engine that is capable of identifying individuals' records efficiently and accurately from just a single facial photo.
\end{abstract}

Index Terms-Privacy, Linkage attacks, Online social networks

\section{INTRODUCTION}

Phone numbers, as evidenced by the wide adoption of 2factor authentication, are reliable tokens for uniquely identifying individuals (users). They are also employed by many social media applications to allow users to identify friends, without having to search for people using full names (or usernames) that may not constitute identifiability across platforms. Searching by phone numbers and contact discovery are such two features that are desirable both to the users and to the services as it makes migration to a platform easier, especially when signing up, increasing the chances of user adoption [1].

*Watch Dogs is a PC game developed by Ubisoft Inc. following a hacker who uses facial search to instantly identify and access information about citizens
Albeit the convenience of contact discovery, large-scale unmonitored use of this feature may result in attackers obtaining corpusses of phone numbers registered to the services along with the accounts they are attributed to. There have been numerous works [2], [3], [5] establishing the risk associated to vast phone number validation, which can lead to phishing attacks and illegal advertising practices, such as robocalling [6].

Using this feature, an attacker can create a massive dataset of active phone numbers (belonging to real individuals) residing in a particular geographic region (e.g., a city or country). Furthermore, by utilizing all personal information from the queried social networks, the attacker can also include several attributes (including face photos, occupation, biography, relationship status, etc.) of the individuals along with their phone numbers into this dataset. One serious consequence of possessing such a dataset is the possibility to link a total stranger (that the attacker comes across in public for example) to one of the records in the dataset via facial search. This is possible if a high fraction of individuals' facial photos and associated records are available. A facial matching capability would allow the attacker to immediately learn a vast amount of personal information from the records about the person, which may have serious consequences, including (but not limited to) discrimination or taking advantage of the person's sensitive information in the dataset (e.g., salary, occupation, or relationship status). This privacy risk can also be seen in the popular computer game Watch Dogs [7], in which the main character takes photos of strangers in public (as shown in Figure 1) to instantly observe their personal information (e.g., occupation or financial records).

In this work, by administrating the introduced exploits we quantify the privacy risk of the aforementioned linkage attack. For this, we first explore and show the practicality of vast phone number validation attacks to WhatsApp (similar to a recent work [5]). Using the validated numbers (through WhatsApp), we utilize our exploits on other popular social networks (such as Facebook and Twitter) and extract up to 23 additional attributes belonging to individuals that own the validated numbers. By doing so, we construct a large dataset 


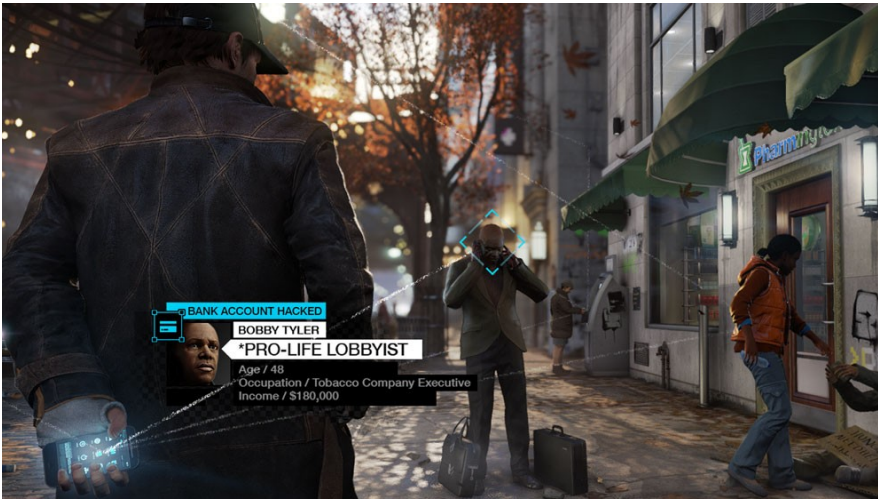

Fig. 1. Profiler from gameplay of Watch Dogs [7] courtesy of Ubisoft Entertainment.

including vast amount of personal information for individuals residing in a given region. Then, we show and quantify the risk of mass scale facial indexing by simulating a linkage attack using a facial photo of a victim (that can be taken in public). A linkage attack here refers to the ability to identify and access the collected records of the victim by matching their facial photo with an entry on the constructed dataset.

The rest of the paper is organized as follows. In the next section, we summarize the related work. In Section III we detail our approach, including dataset construction and the identified vulnerability. In Section IV, we conduct tests on constructed datasets and report our results. In Section V, we discuss some simple countermeasures. Finally in Section VI, we conclude the paper.

\section{RELATED WORK}

Several works in the literature have explored privacy risks associated with contact discovery features across mobile messaging applications, starting with Cheng et al. [2]. The creator of Signal, Moxie Marlinspike, expressed privacy-preserving contact discovery as an unresolved problem [1]. With the more recent adoption of two-factor authentication and contact discovery features moving beyond messaging applications, another line of work by Gupta et al. [3] represented phone numbers as "Personally Identifiable Information", stating them as the most vulnerable attack vector to individuals. Gupta et al. [4] also showed practical vast targeted phishing attacks were plausible with phone numbers. More recent consequences of this threat also include exponential rise of robocalls across US [6]. Kim et al. [22] demonstrated that phone number verification attacks are possible to Facebook with limited data collection. Over time, as contact discovery has been more widely adopted, social media platforms introduced more rate limitations to mitigate attacks due to this attribute [10], [11]. A recent paper by Hagen et al. [5] showed that vast phone number validation attacks for major mobile messengers are still possible with little resources using easily created registries of phone numbers and hash reversal techniques. One common thing about most of these aforementioned approaches is that they establish the risks associated with contact discovery by the ability to collect corpuses of phone numbers registered to the social media services. However, they do not crossreference and index exposed data attributes across platforms. Our contributions allow for much more and sensitive data points to be collected while also compromising arguably the most consequential personally identifiable information, our faces.

\section{Methodology}

We demonstrate how an attacker may amass compromising data about individuals by utilizing exploits on certain social media platforms with a reasonable amount of computing resources and without the knowledge of neither the exploited services nor the victims. In the following, we describe the details of our methodology, including dataset construction and the considered linkage attack (of random photos of individuals to their records in the constructed dataset).

\section{A. Dataset Construction}

Constructing the dataset consists of two stages. First, we exhaustively generate and validate phone numbers in bulk using WhatsApp. Then, we search social media platforms (Facebook Messenger and Twitter) for matching accounts of the owners of each phone number. As phone numbers are unique and cannot be faked, we assume the authenticity of discovered accounts.

1) Phone Number Generation and Validation: A dataset of active phone numbers is a requirement to employ contact discovery-based attacks on social media platforms. To address this issue, we use phone prefixes for a country (where we conduct the attack) and generate all the possible phone number combinations. The reason we choose WhatApp as the starting point is the popularity of this platform in our target population (individuals in Turkey), in which a significantly high fraction of population is WhatsApp users.

By exploiting the low rate limitation for contact discovery, in a viable time-frame and with limited computational resources, we systematically validated all of our generated phone numbers (i.e., verified whether they have a WhatsApp account) and stored the matching numbers. We used WhatsApp's contact discovery API for our number validation part, which is exposed to apps such as "Contacts" (native to Android phones) to notify users about whether a contact added is associated with WhatsApp. Using this method, automated phone number validation was viable until February 2020, after which daily rate limitations were introduced (we conducted our data collection before this date). However, we discovered a new exploit utilizing "Dual Apps" feature on Android phones, which allows for 2 independent instances of WhatsApp on a single device. One can use this exploit to exhaust limitations on a single instance, deregister the account, and change to the second instance to register again with the same credentials [19]. Following this procedure, we observed a reset on the rate limitations. Therefore, one can continue to interchange between "Dual" WhatsApp instances with no registry caps, 
allowing for vast phone number validation. To justify this, using this new exploit, we validated more than $60 \mathrm{k}$ phone numbers a day on a single device. Our findings were reported to Facebook as part of the Bug Bounty Program on February 2020.

Statistical results for our phone number generation and validation for a given region are shown in Table I. We observed that one in every 3.57 phone number $(300 \mathrm{M} / 84 \mathrm{M})$ is actively in use [8], [9], which marks the bar for the fraction of numbers (that belong to real individuals) we can discover from randomly generated phone numbers. Indeed, we observed that one in every 4.41 randomly generated phone number (200K/45K) belongs to a valid WhatsApp account, which shows that (i) our discovery rate is very close to the bar and (ii) more than $80 \%$ of the target population are WhatsApp users.

TABLE I

STATISTICAL RESULTS FOR PHONE NUMBER GENERATION AND VALIDATION.

\begin{tabular}{cc}
\hline \hline Theoretical limit for total number of phone numbers [8] & $300 \mathrm{M}$ \\
Number of phone numbers in use [9] & $84 \mathrm{M}$ \\
Number of phone numbers generated at random & 200,000 \\
Number of phone numbers validated on WhatsApp & 45,349 \\
Projected percentage of WhatsApp users for region & $80.9 \%$ \\
\hline
\end{tabular}

Alternatively, one might consider using Facebook's directory [18] and crawling through every Facebook profile page to generate a dataset of profiles, instead of going through the phone number generation phase. Even assuming crawling large amount data from Facebook profile directory is possible (which is non-trivial due to Facebook's anti-crawling tools), the profiles one can collect this way are only limited to Facebook and (unless other social media accounts are provided by the user on their profile) are not directly linkable to other accounts of the target. Furthermore, implementations, such as [21] which can be used to conduct facial searches and other queries across platforms are not plausible, as they cannot provide real-time search capabilities and they require human operators to validate results.

2) Collection of Attributes: After systematically obtaining the list of WhatsApp users by their phone numbers, we examined social media platforms using either "phone number search" or "contact discovery" features to match the verified phone numbers with their affiliated accounts and to extract additional data attributes about individual users. We associated the numbers with the specific social media account they map to, thus we had to process each number one at a time. We chose to target widely adopted services, such as Facebook Messenger and Twitter for this task, as they had similar low rate limits for contact discovery via phone number (during the time of our study). It is worth noting that these exploits have since then came to Facebook's attention and they have removed phone number search features both from Facebook
Messenger and later from its lite version as of April 2019 [10]. Similarly, Twitter also increased rate limits for its contact discovery feature after a researcher crawled 17 million phone numbers in December 2019 [11]. As discussed, our procedures were not affected, since data collection was completed before these security patches. Overall, the attributes that are available for collection using our exploits on Facebook, WhatsApp, and Twitter, which we seek to collect for each individual in a given region, are shown in Table II (depending on what the individual shares on their profile, we collect a subset of these attributes). We also provide the flow diagram for dataset construction in Figure III-A2.

TABLE II

SOME OF THE COLLECTED ATTRIBUTES OF INDIVIDUALS (DATA POINTS RELATED TO THE SAME ATTRIBUTES ARE JOINED).

\begin{tabular}{lccc}
\hline \hline Attributes & WhatsApp & Facebook M. & Twitter \\
\hline Profile URL & $x$ & $\checkmark$ & $\checkmark$ \\
User Handle & $x$ & $\checkmark$ & $\checkmark$ \\
Full Name & $x$ & $\checkmark$ & $\checkmark$ \\
Profile Picture & $\checkmark$ & $\checkmark$ & $\checkmark$ \\
Photos & $x$ & $\checkmark$ & $x$ \\
Date of Birth & $x$ & $\checkmark$ & $\checkmark$ \\
Biography & $x$ & $x$ & $\checkmark$ \\
Location(s) & $x$ & $\checkmark$ & $\checkmark$ \\
Check-in(s) & $x$ & $\checkmark$ & $x$ \\
Occupation(s) & $x$ & $\checkmark$ & $x$ \\
Education & $x$ & $\checkmark$ & $x$ \\
Gender & $x$ & $\checkmark$ & $x$ \\
Interests & $x$ & $\checkmark$ & $x$ \\
Languages & $x$ & $\checkmark$ & $x$ \\
Religious View & $x$ & $\checkmark$ & $x$ \\
Affiliated URLs & $x$ & $\checkmark$ & $\checkmark$ \\
URLs to Friends & $x$ & $\checkmark$ & $x$ \\
URLs to Family & $x$ & $\checkmark$ & $x$ \\
\hline
\end{tabular}

To collect data, we exploited Facebook Messenger and Twitter's "contact discovery" features by using UI automation. For this, we used test automation software, such as Tasker [15] and Selenium [17], which simulate human interaction. In practice, such a data collection technique is not preferred (compared to API-based techniques) since it is significantly slower and more prone to errors as UI elements change. In particular, Facebook is notoriously difficult to crawl with UI automation because of regular updates on the user experience. However, at the time of our study, there was no exposed exploitable API discovered for neither of the platforms, and hence we crawled both using UI automation.

Filtering the number of phone numbers to be searched by only using the numbers validated through WhatsApp drastically increased the speed of data collection. Our proof-ofconcept tests for data collection (concluded before the updates on rate limitation) took around 45 seconds to fully collect Twitter, Facebook, and WhatsApp profiles of a user using an off-the-shelf Android phone. Parallelizing these tasks across 
phones can vastly reduce the total data collection time by eliminating app switching overhead. In theory, one can complete extraction of 50,000,000 profiles with 300 automatons in 90 days. Android emulators are also shown to be feasible for this [5].

3) Pre-processing Data: Before evaluation we preprocessed all incoming data for indexing and fast facial search. Using OpenCVs facial detection [13] tool, we removed the duplicates, cropped, and saved any image data that include faces. Cropped faces were uploaded to Face++ [16], which annotates and stores them for easier search via the Facial Search API [14]. After annotation, a facial search within the API returns a match in milliseconds regardless of dataset size. We also removed all the images that do not contain any faces for privacy reasons. Note that despite we, not directly utilizing them, photos that do not contain any faces still constitute a valuable source of intelligence. For instance, a high fraction of photos we collected contained an Arabic word, which means God and this provides valuable information about religious beliefs of the users. To gain such information from digital images, one would need to run OCR (Optical Character Recognition) and object recognition algorithms on every collected photo, which would require a high amount of processing power. However, we observed that Facebook already stores this sort of information as metadata embedded inside each photo uploaded to their platform. We plan to explore such information for the identified vulnerability in future work.

\section{B. Facial Search in the Dataset}

As discussed, we consider the privacy risk due to the attacker first generating a large dataset (using methods in Sections III-A1 and III-A2), and then conducting facial searches. We consider the case, where the attacker takes a photo of a random individual (victim) in public, and then tries to identify the record of the victim in its constructed dataset. This is possible if a facial photo of the victim has been obtained through our exploits. In the case of a match, the attacker will access the victims records which potentially include 23 other data points, as described in Table II. Even without such a linkage attack, an attacker being able to index this many sensitive attributes about individuals at mass scale, without detection and relatively moderate computation power is significant. To create a close to real-life scenario, we handpicked a "viable" face from each phone numbers' corpus of faces and separated the picked photo to a separate pool. We simulated our considered scenario using such a method (rather than actually taking an instant photo of a potential victim) because we did not have physical access to any of the individuals in the constructed dataset. We also decided against choosing a random photo for each phone number because for a fair amount of the phone numbers, we observed faces that does not belong to the actual individual (e.g., faces of national figures or famous people).

\section{Evaluation}

We implemented our phone number generator on Android by utilizing Whatsapp's Contacts API to continuously extract registered phone numbers for a given state. The Facebook and Twitter profiles linked to the verified phone numbers are obtained through contact discovery features on Facebook Messenger and Twitter (both Android apps), which we exploited via UI automation. Gathered social media links are passed to a Selenium-based crawler for Twitter and Facebook, which collects most of the data points on the profile pages.

While preparing our photos for the testing phase, we first used OpenCV [13] for face detection, and then passed the detected faces to a cloud-based solution, Face++ [16], to conduct search by using its facial recognition feature.

For the evaluation, we used 1457 phone numbers that were chosen indiscriminatelyamong the generated and verified numbers collected using the WhatsApp Contacts exploit (as stated in Section III-A1). Their corresponding data attributes (as shown in Table II) were gathered using the Facebook and Twitter exploits (as in Section III-A2). Among the individuals in the constructed dataset, we observed an average of 3.94 attributes per person (excluding phone numbers). Also, 57\% (833) of the individuals in the constructed dataset had at least 1 facial photo (details are in Table III). However, to comply with our test scenario we only conducted tests on people to whom more than one face was associated to (525).

TABLE III

STATISTICS ABOUT THE EVALUATION OF THE LINKAGE ATTACK.

\begin{tabular}{lc}
\hline \hline Number of phone numbers used & 1457 \\
Phone numbers, for which there is at least one face associated & 833 \\
Number of viable candidates for test scenario & 525 \\
Average number of photos extracted per phone number & 8.68 \\
Average number of faces detected per phone number & 8.20 \\
\hline
\end{tabular}

Overall, we observed the accuracy of the identified attack as $67 \%$. In other words, from the set of 525 phone numbers we conducted our test, 355 were correctly identified by facial search, 41 were falsely matched, and 129 of them returned "inconclusive" from the facial recognition. Overall, we observed that the attacker, with high accuracy, can identify the detailed record of a random person (for which the attacker only has one facial photo) in its constructed dataset. We expect to have significantly better accuracy if we were to pair the considered attack with a state-of-the-art facial detection and recognition software along with a real-life scenario, in which we could have used photos taken in the wild rather than substituting from the collected data.

In the constructed dataset, we collected 24 different attributes, for which the coverage is shown in Table IV. We observed that the top 5 most collected attributes are full name, affiliated URLs, faces and other photos, gender, and location. As we show in Figure 3, more than $42 \%$ of the people in our constructed dataset has at least 5 attributes (which is 


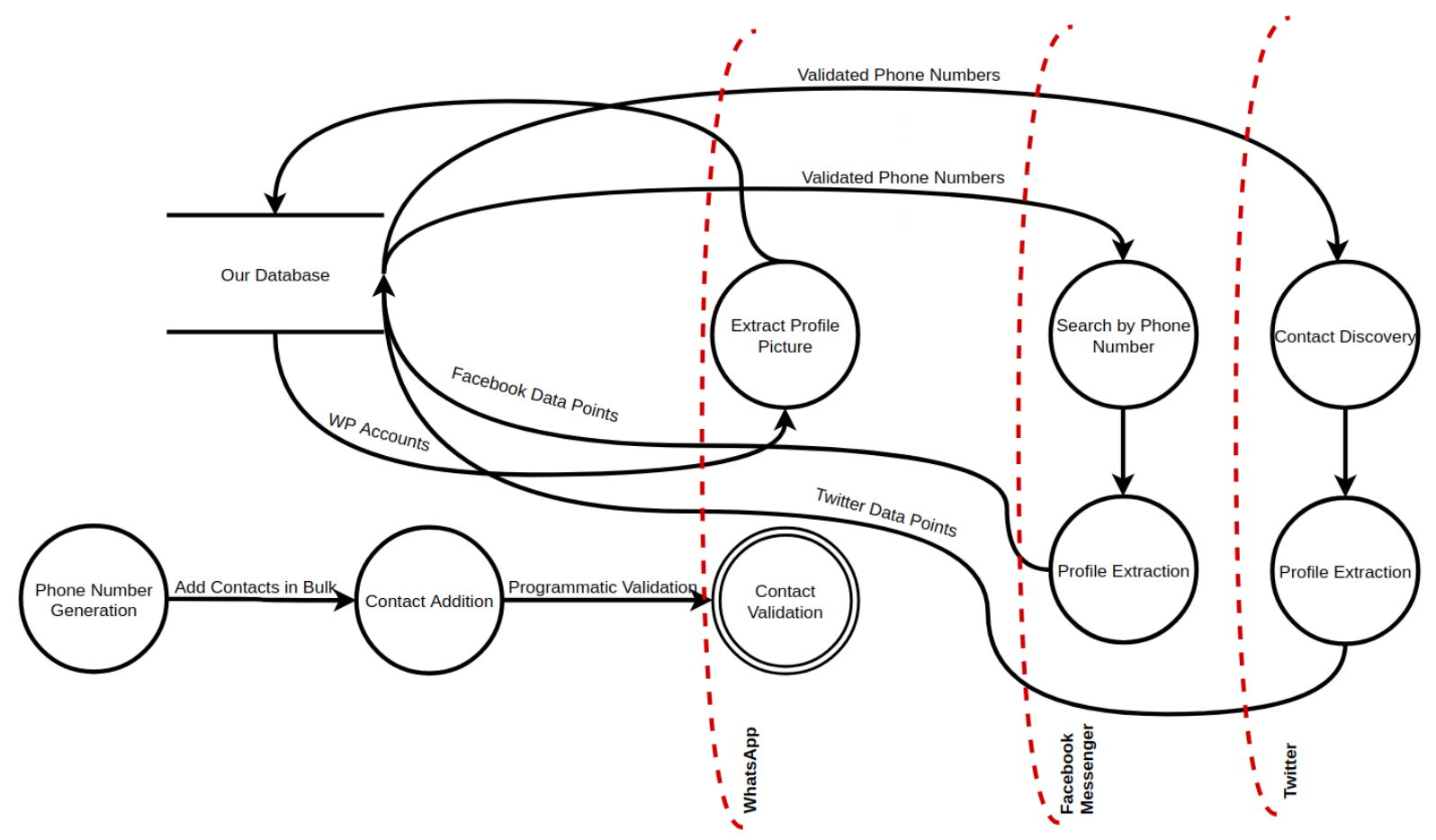

Fig. 2. Flow diagram for dataset construction.

typically sufficient for cross-platform identification). Thus, the attacker can first learn such attributes about the victims from the constructed dataset, and then use such attributes to identify more accounts of the victims across other platforms.

TABLE IV

DETAILED RESULTS OF THE LINKAGE ATTACK (DATA POINTS RELATED TO THE SAME ATTRIBUTES ARE JOINED).

\begin{tabular}{lcc}
\hline \hline & Out of 1457 & Coverage \\
\hline Number of Facebook accounts & 726 & $49.83 \%$ \\
Number of Twitter accounts & 213 & $14.62 \%$ \\
Full Name & 774 & $53.12 \%$ \\
Location(s) & 440 & $30.20 \%$ \\
Occupation(s) & 229 & $15.71 \%$ \\
Education & 266 & $18.26 \%$ \\
Affiliated URLs & 513 & $35.21 \%$ \\
URLs to Family & 88 & $6.03 \%$ \\
Interests & 47 & $3.20 \%$ \\
Religious View & 19 & $1.30 \%$ \\
Gender & 631 & $43.31 \%$ \\
Date of Birth & 74 & $5.08 \%$ \\
Languages & 71 & $4.87 \%$ \\
Faces from Facebook & 620 & $42.55 \%$ \\
Friends & 253 & $17.36 \%$ \\
Twitter bio & 72 & $4.94 \%$ \\
Twitter photo & 117 & $8.03 \%$ \\
WhatsApp photo & 446 & $30.61 \%$ \\
Check-ins & 0 & $0 \%$ \\
\hline
\end{tabular}

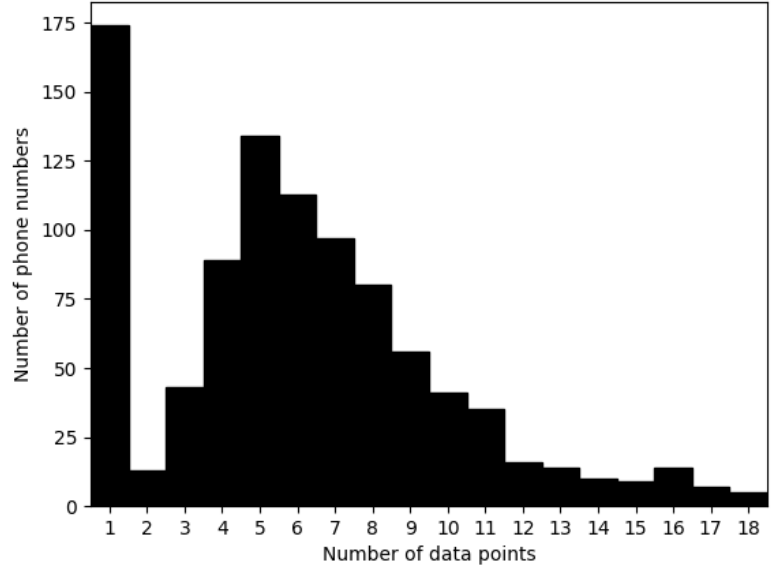

Fig. 3. Distribution of number of data attributes collected

\section{Countermeasures}

With many social media apps integrating contact synchronization (Facebook Messenger, Twitter, WhatsApp, Instagram, etc.) or even phone number search (Facebook Messenger had this feature until April 2019) as a means of accustoming a new user with their friends, we come across a tradeoff between privacy and practicality. Having examined 11 (WhatsApp, Swarm, Foursquare, Line, Twitter, Facebook Messenger, Facebook Messenger Lite, Snapchat, Instagram, Viber, and LinkedIn) popular social media apps over the course of 3 years, we have put together the following simple 
recommendations to mitigate the identified vulnerability:

1) Platforms can introduce stricter rate limits on contact discovery and enforce different rate limits for professional versus personal accounts. This would mitigate programmatic validation attacks.

2) Adding notification upon discovery by phone number (similar to LinkedIn profile visit notifications) could inform users about who looked them up, and hence users can report suspicious activity. This in return can help platforms identify unusual activity.

3) Use of selective contact permissions (as stated in [5]) that allow users to sync only a limited portion of their contacts can deter users from uploading their full address books to social media platforms.

4) Removing search by phone number features (like Facebook Messenger recently did [10]) can nullify phone number look-ups by search. Furthermore, a new data security paradigm focused on protecting user privacy with tokens that are not personally identifiable across other platforms can entirely remove phone numbers as a vector of attack as well as reducing risk of exposure if discovered on a single platform. This idea has recently been pioneered by Signal [20].

\section{DiscuSSION AND CONCLUSION}

Despite the convenience of contact discovery, there are risks associated with unmonitored usage of this feature across platforms. We have shown that an attacker with a moderate amount of computing resources can amass a nationwide dataset on citizens without being traced. The content of constructed dataset includes sensitive personally identifiable information [3], such as phone numbers and facial data (shown in Table IV). This provides the attacker with an unprecedented capability of accessing detailed records of a person in mere milliseconds using only a single facial photo much like a technological dystopia. Our evaluation on a test dataset reveals that such an attack is viable for more than a third of individuals (Section IV) residing in a particular region. Thus, our findings, although on a relatively small dataset, show the significance of an important privacy risk.

The consequences of being able to utilize a few hundred parallelized virtual machines to collect mass data on citizens are massive. One recent example being Cambridge Analytica, where email addresses were used to illegally identify potential audiences across platforms and deliver personalized political ads [12]. In contrast, our work focuses on the ultimate identifiable token, the human face. Such technology, if commercialized or used maliciously, could essentially result in our faces becoming tradeable "cookies" at the expense of any entity, eradicating any hope of privacy in the modern era of information age.

\section{REFERENCES}

[1] M. Marlinspike, "The Difficulty of Private Contact Discovery," signal.org, Jan. 3, 2014. [Online]. Available: https://signal.org/blog/contactdiscovery/. [Accessed: Oct. 25, 2020].
[2] Y. Cheng, L. Ying, S. Jiao, P. Su, and D. Feng, "Bind Your Phone Number with Caution: Automated User Profiling Through Address Book Matching on Smartphone," in ASIA CCS '13: 8th ACM Symposium on Information, Computer and Communications Security, Hangzhou, China, May 2013, K. Chen, Q. Xie, and W. Qiu, Association for Computing Machinery, New York, NY, United States, 2013.

[3] S. Gupta, "Emerging Threats Abusing Phone Numbers Exploiting CrossPlatform Features," in International Conference on Advances in Social Network Analysis and Mining, ASONAM, San Francisco, CA, USA, August 18-21, 2016, IEEE, 2016. pp. 1339-41.

[4] S. Gupta, "Exploiting phone numbers and cross-application features in targeted mobile attacks," in Proceedings of the 6th Workshop on Security and Privacy in Smartphones and Mobile Devices, 2016, 73-82

[5] C. Hagen, C. Weinert, C. Sendner, A. Dmitrienko, and T. Schneider, "All the Numbers are US: Large-scale Abuse of Contact Discovery in Mobile Messengers", NDSS. Internet Society, vol. 2021, September 2020.

[6] S. M. Kelly, "The frightening future of robocalls: Numbers and voices you know," CNN, April 6, 2019. [Online]. Available: https://edition.cnn.com. [Accessed: Oct. 16, 2020].

[7] Ubisoft Entertainment SA, "Watch Dogs," ubisoft.com. [Online]. Available: https://www.ubisoft.com/en-us/game/watch-dogs/watch-dogs. [Accessed: Nov. 11, 2020].

[8] BTK Information and Communication Technologies Authority, Numara Yönetim Sistemi Numaralandırma Raporu (Kamuya Açı) [A Numbering Report of Number Management System (Open to Public)]. November 11, 2020. [Online]. Available: https://www.btk.gov.tr/uploads/ntsfiles/NPR.pdf. [Accessed: Nov. $13,2020]$.

[9] BTK Information and Communication Technologies Authority, Türkiye Elektronik Haberleşme Sektörü 3 Aylık Pazar Verileri Raporu [Turkey Electronic Communications Sector Three-Month Market Data Report]. 2020. [Online] Available: https://www.btk.gov.tr/uploads/pages/pazarverileri/turkiye-haberlesme-raporu-002.pdf. Accessed: Nov. 11, 2020.

[10] Z. Whittaker, "Facebook Won't Let You Opt Out of Its Phone Number 'Look up' Setting," https://techcrunch.com March 4, 2019. [Online]. Available: https://techcrunch.com/2019/03/03/facebook-phonenumber-look-up/. [Accessed: Oct. 30, 2020].

[11] Z. Whittaker, "A Twitter App Bug Was Used to Match 17 Million Phone Numbers to User Accounts," https://techcrunch.com December 24, 2019. [Online]. Available: https://techcrunch.com/2019/12/24/twitter-androidbug-phone-numbers/. [Accessed: Oct. 30, 2020].

[12] D. Ghosh, and B. Scott, "Facebook's New Controversy Shows How Easily Online Political Ads Can Manipulate You," TIME, March 19, 2018. [Online]. Available: https://time.com/5197255/facebookcambridge-analytica-donald-trump-ads-data/. [Accessed: Nov. 8, 2020].

[13] Open Source Computer Vision, "Cascade Classifier," opencvorg. [Online]. Available: https://docs.opencv.org/3.4/db/d28/tutorial_cascade_classifier.html. [Accessed: Nov. 8, 2020].

[14] Megvii, "Face Searching," faceplusplus.com. [Online]. Available: https://www.faceplusplus.com/face-searching/. [Accessed: Nov. 12, 2020].

[15] Tasker, "Total Automation for Android," taskerjoaoapps.com. [Online]. Available: https://tasker.joaoapps.com. [Accessed: Nov. 8, 2020].

[16] "FaceSet Create API", https://console.faceplusplus.com. [Online]. Available: https://console.faceplusplus.com/documents/6329329. [Accessed: Oct. 30, 2020].

[17] "Selenium Automates Browsers. That's it!", selenium.dev. [Online]. Available: https://www.selenium.dev/. [Accessed: Nov. 12, 2020].

[18] "People Directory Results for A - azzyk'Newbie", facebook.com. [Online]. Available: https://www.facebook.com/directory/people/. [Accessed: Sept. 27, 2020].

[19] "What is Dual Messenger?", samsung.com. [Online]. Available: https://www.samsung.com/global/galaxy/what-is/dual-messenger/. [Accessed: Sept. 28, 2020]

[20] "Introducing Signal PINs", signal.org. [Online]. Available: https://signal.org/blog/signal-pins/. [Accessed: Nov. 5, 2020]

[21] "Social Mapper", github.com. [Online]. Available: https://github.com/Greenwolf/social_mapper. [Accessed: Dec. 1, 2020]

[22] J. Kim, "Hello, Facebook! Here is the stalkers' paradise!: Design and analysis of enumeration attack using phone numbers on Facebook," in International Conference on Information Security Practice and Experience, Springer, 2017. pp. 663-77. 